\title{
molecules
}

ISSN 1420-3049

www.mdpi.com/journal/molecules

Article

\section{The in Vitro Structure-Related Anti-Cancer Activity of Ginsenosides and Their Derivatives}

\author{
Hang Dong ${ }^{1, \dagger}$, Li-Ping Bai ${ }^{1,2, \dagger}$, Vincent Kam Wai Wong ${ }^{1,2}$, Hua Zhou ${ }^{1,2}$, Jing-Rong Wang ${ }^{1,2}$, \\ Yan Liu ${ }^{1}$, Zhi-Hong Jiang ${ }^{1,2, *}$ and Liang Liu ${ }^{1,2, *}$
}

1 Shum Yiu Foon Shum Bik Chuen Memorial Centre for Cancer and Inflammation Research,

School of Chinese Medicine, Hong Kong Baptist University, 7 Baptist University Road, Kowloon Tong, Hong Kong, China

2 State Key Laboratory of Quality Research in Chinese Medicine, Macau University of Science and Technology, Avenida Wai Long, Taipa, Macau, China

$\dagger$ These authors contributed equally to this work.

* Authors to whom correspondence should be addressed; E-Mails: zhjiang@must.edu.mo (Z.-H.J.); 1liu@must.edu.mo (L.L.); Tel.: +853-8897-2799; Fax: + 853-2882-7222.

Received: 25 October 2011; in revised form: 9 December 2011 / Accepted: 16 December 2011 / Published: 19 December 2011

\begin{abstract}
Panax ginseng has long been used in Asia as a herbal medicine for the prevention and treatment of various diseases, including cancer. The current study evaluated the cytotoxic potency against a variety of cancer cells by using ginseng ethanol extracts (RSE), protopanaxadiol (PPD)-type, protopanaxatriol (PPT)-type ginsenosides fractions, and their hydrolysates, which were prepared by stepwise hydrolysis of the sugar moieties of the ginsenosides. The results showed that the cytotoxic potency of the hydrolysates of RSE and total PPD-type or PPT-type ginsenoside fractions was much stronger than the original RSE and ginsenosides; especially the hydrolysate of PPD-type ginsenoside fractions. Subsequently, two derivatives of protopanaxadiol (1), compounds $\mathbf{2}$ and 3, were synthesized via hydrogenation and dehydration reactions of compound $\mathbf{1}$. Using those two derivatives and the original ginsenosides, a comparative study on various cancer cell lines was conducted; the results demonstrated that the cytotoxic potency was generally in the descending order of compound $3>20(S)$-dihydroprotopanaxadiol (2) $>$ PPD (1) $>20(S)$ $\mathrm{Rh} 2>20(R)-\mathrm{Rh} 2 \approx 20(R)-\mathrm{Rg} 3 \approx 20(S)-\mathrm{Rg} 3$. The results clearly indicate the structurerelated activities in which the compound with less polar chemical structures possesses higher cytotoxic activity towards cancer cells.
\end{abstract}


Keywords: ginsenosides; anti-cancer; cytotoxicity; protopanaxadiol; structure-related activity

\section{Introduction}

Cancer, a generic term for a large group of diseases that can affect any part of the body, is a leading cause of death worldwide and accounted for 7.6 million deaths (around 13\% of all deaths) in 2008 [1]. The major interventions of conventional medicine include surgery and radio/chemotherapy which aim at eliminating the cancer or prolonging life span of the patients [2]. However, it is often of those interventions failed to reach their expected results. Thus, patients, doctors, and researchers are looking elsewhere for effective therapeutics, particularly from natural sources [3].

Panax ginseng has become, arguably, the most popular herb for treatment of various diseases, including cancers, in Asia if not across the World. Ginseng extracts and its chemical components have been pronounced capable of reducing risk of cancers, including inhibition of carcinogenesis in oral cavity, stomach, lung, liver, pancreas, ovaries, and colon [4]. The pharmacological properties of ginseng are generally attributed to its component triterpene glycosides, called ginsenosides [5]. Nevertheless, whether the intact ginseng is suitable for in vitro anticancer studies and which hydrolysis products could exert anticancer effect remains unclear. Previous reports showed that the degradation and metabolism of glycosides derived from medical plants often affect their pharmacological potency as well as their clinical therapeutic efficacy; these degradation and metabolism processes occur even in the gastrointestinal tract [6]. For instance, the ginsenosides $\mathrm{Rb} 1, \mathrm{Rb} 2, \mathrm{Rc}, \mathrm{Re}$ can be hydrolyzed in artificial gastric fluid (at $37^{\circ} \mathrm{C}$ and $\mathrm{pH}$ 1.2) [7,8]; this could alter the composition of ginsenosides as well as their corresponding bioactivities both qualitatively and quantitatively [9,10]. It is estimated that the hydrolytes and/or metabolites of ginsenosides may play an important role in anti-cancer in vivo when ginsenosides are taken orally. Therefore, we screened the bioactive anti-cancer compounds from ginseng by using hydrolytes of ginseng extracts and ginsenosides rather than using their original extracts and ginsenosides. We expected that using hydrolyzed products of ginseng would more closely mimic the biochemistry of ginseng in patients, and thus results would be more relevant to clinical usage.

Depending on their aglycone content, ginsenosides are divided into protopanaxadiol (PPD)-type, protopanaxatriol (PPT)-type and oleanane-type ginsenosides. As ginsenosides are subject to metabolism mainly via hydrolysis and dehydration processes in the gastrointestinal tract, we therefore evaluated the cytotoxicity of ginseng extract and ginsenosides fractions after a stepwise hydrolysis of the sugar moieties against a variety of cancer cells. To further elucidate the activity-structure relationship of ginsenosides, we synthesized two derivatives (compounds 2 and 3) of PPD (1) and evaluated their cytotoxic effects on a series of cancer cell lines. As a result, these two derivatives 2, 3 derived from PPD (1) were, for the first time, found to have potent anticancer potency, and of these compound 3 demonstrated the highest anticancer activity. 


\section{Results and Discussion}

\subsection{Hydrolysate of Ginseng Extracts Preparation and Its Cytotoxicity}

The ethanol extract of ginseng (RSE) was hydrolyzed in artificial gastric juice to prepare the hydrolysates of RSE (RSEH) for the study. Briefly, the dried RSE (20 g) was dissolved in the artificial gastric juice $(0.1 \mathrm{~N} \mathrm{HCl}, 1,800 \mathrm{~mL})$ and then subjected to incubation in a VK7025 Dissolution Tester for $2 \mathrm{~h}$ (Temp: $37 \pm 0.5{ }^{\circ} \mathrm{C}$; Sprindle speed: $100 \mathrm{rpm}$ ). The hydrolysis solution was subsequently applied to freeze-dry after being neutralized with $10 \mathrm{M} \mathrm{NaOH}$ to yield RSEH (32.7 g, the content of $\mathrm{NaCl}$ is $38.8 \%$ ). The composition and contents of RSEH were analyzed by HPLC-TOF-MS. By comparing the retention times with authentic compounds, primary and secondary ginsenosides (de-glycosylated ginsenosides) could be identified (Figure 1). The results showed that the secondary ginsenosides such as Rg3, Rk1 and Rg5 accounted for the majority of the constituents in RSEH.

Figure 1. LC-UV chromatograms of the RSE hydrolysates (RSEH).

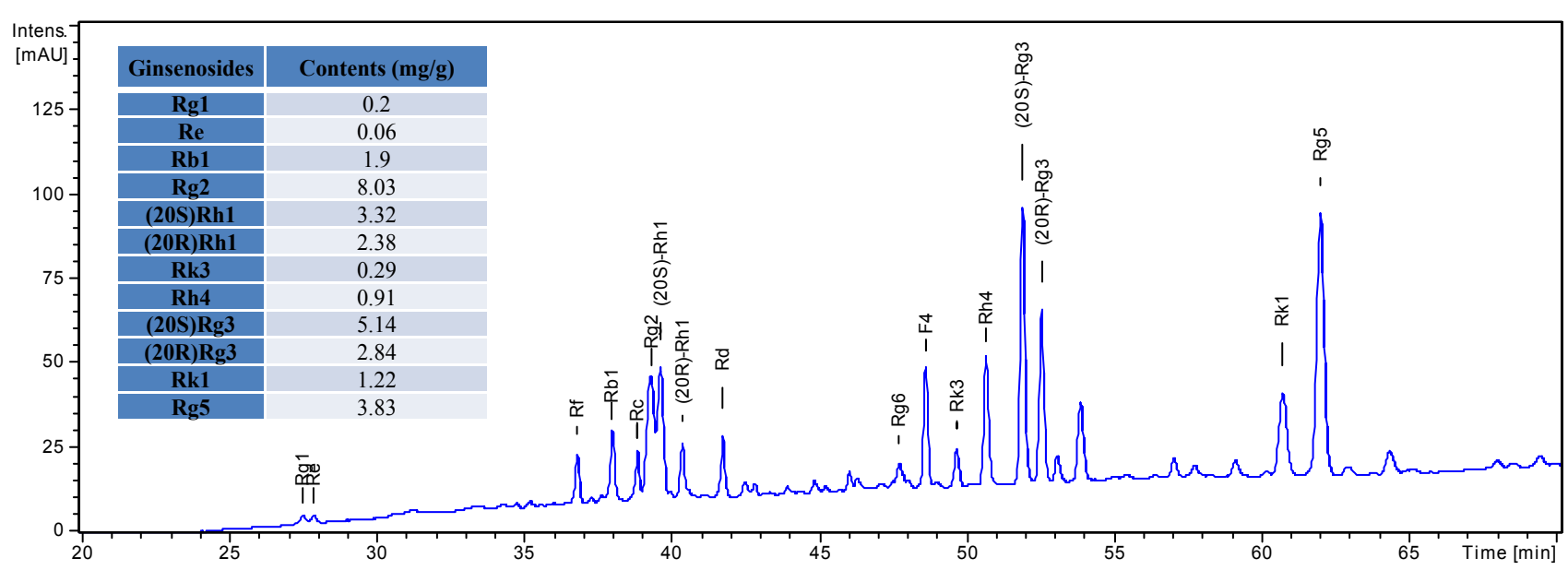

The cytotoxic potency of RSEH and RSE against LLC1 cells with different drug concentrations and treatment time points was compared. Significant and dose-dependent cytotoxicity was observed when LLC1 cells were exposed to RSEH or RSE for 24 to $72 \mathrm{~h}$. The results showed that RSEH exhibited higher cytotoxic effects than RSE after 24 and $48 \mathrm{~h}$, but when LLC1 cells were treated with RSEH or RSE for $72 \mathrm{~h}$, their cytotoxic effects were reversed, i.e., RSE demonstrated much stronger cytotoxic effect than RSEH (Figure 2). This could be explained by the fact that RSEH may be not as stable as the RSE form in the cell culture medium condition, and as a result, the cancer cells might be able to metabolize or detoxify the RSEH after $48 \mathrm{~h}$ treatment $(\mathrm{t}=48 \mathrm{~h})$. In the case of the longer treatment period, e.g., $(\mathrm{t}=72 \mathrm{~h})$, the remaining cancer cells may start to proliferate again and show increased cell viability after $24 \mathrm{~h}$ during the $72 \mathrm{~h}$ MTT assay, and as a result, the cytotoxic effect of RSEH at $72 \mathrm{~h}$ seem to be weaker compared to its cytotoxic effect at $48 \mathrm{~h}$. 
Figure 2. Time- and dose-dependent cytotoxicity of RSEH and RSE on LLC1 cells.
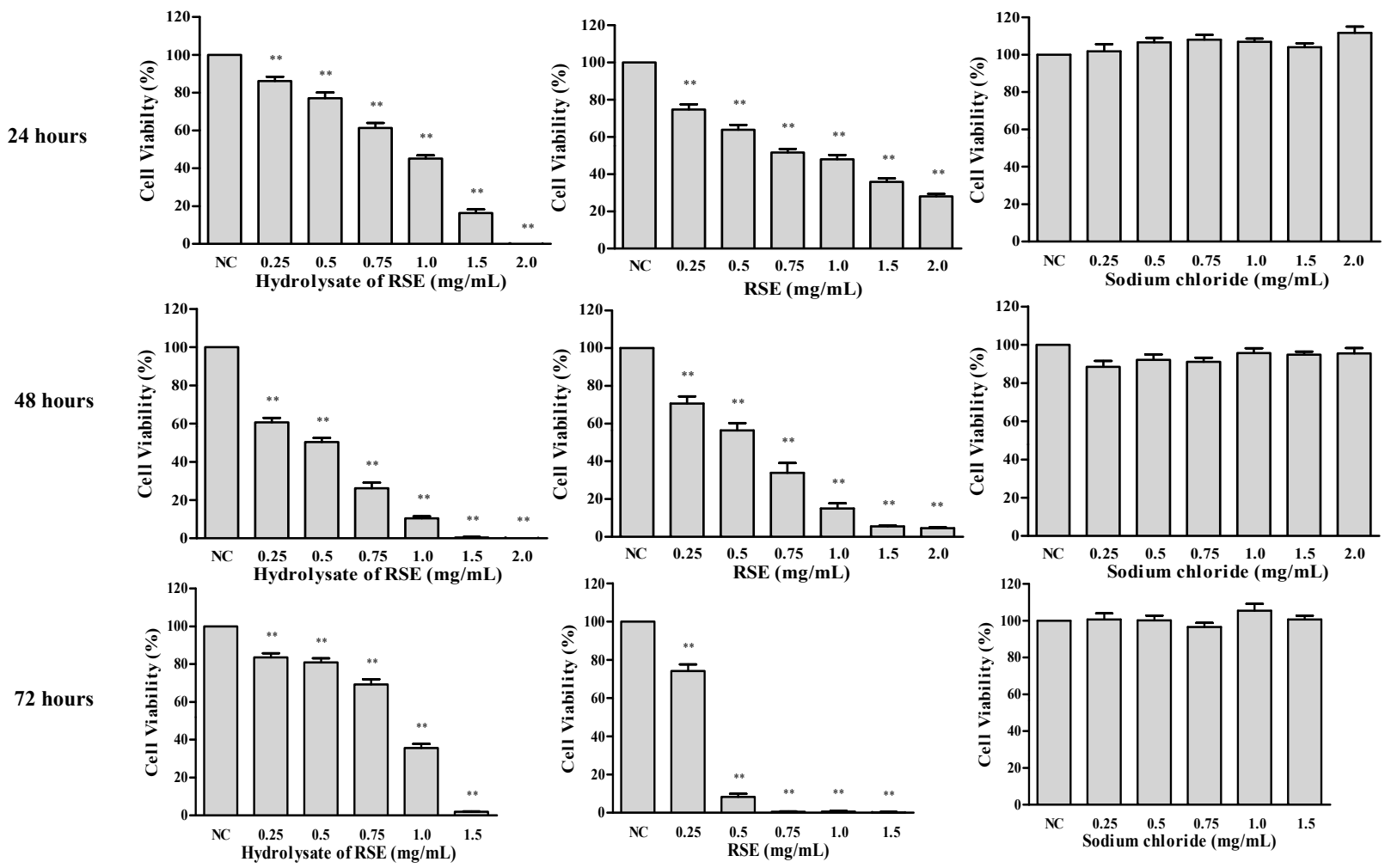

\subsection{Hydrolysate of PPD- or PPT-Type Ginsenosides Fraction Preparation and Its Cytotoxicity}

The PPD- or PPT-type ginsenosides fraction prepared from RSE $(250 \mathrm{mg})$ were dissolved in 300 and $100 \mathrm{~mL}$ artificial gastric acid $(0.1 \mathrm{~N} \mathrm{HCl})$ and incubated in a Julabo Shake Temp SW 22 circulating water bath at $37.5{ }^{\circ} \mathrm{C}$. The hydrolysate solutions were neutralized with $\mathrm{NaOH}$ and subsequently subjected to liquid-liquid extraction with $n$-BuOH $(45 \mathrm{~mL} \times 3)$. The $n$-BuOH extracts were evaporated under reduced pressure, and the residues were freeze-dried to yield hydrolysates of the PPD-type ginsenosides fraction (PPDH, $167 \mathrm{mg}$ ) and the PPT-type ginsenosides fraction (PPTH, $150 \mathrm{mg}$ ). Typical LC-UV chromatograms of PPDH and PPTH are shown in Figure 3. Contents of twelve ginsenosides in the PPDH and PPTH were further determined by using an HPLC-UV method.

Figure 3. HPLC chromatograms of the PPD-type ginsenosides fraction (A) and its hydrolysate PPDH (B), PPT-type ginsenosides fraction (C) and its hydrolysate PPTH (D).

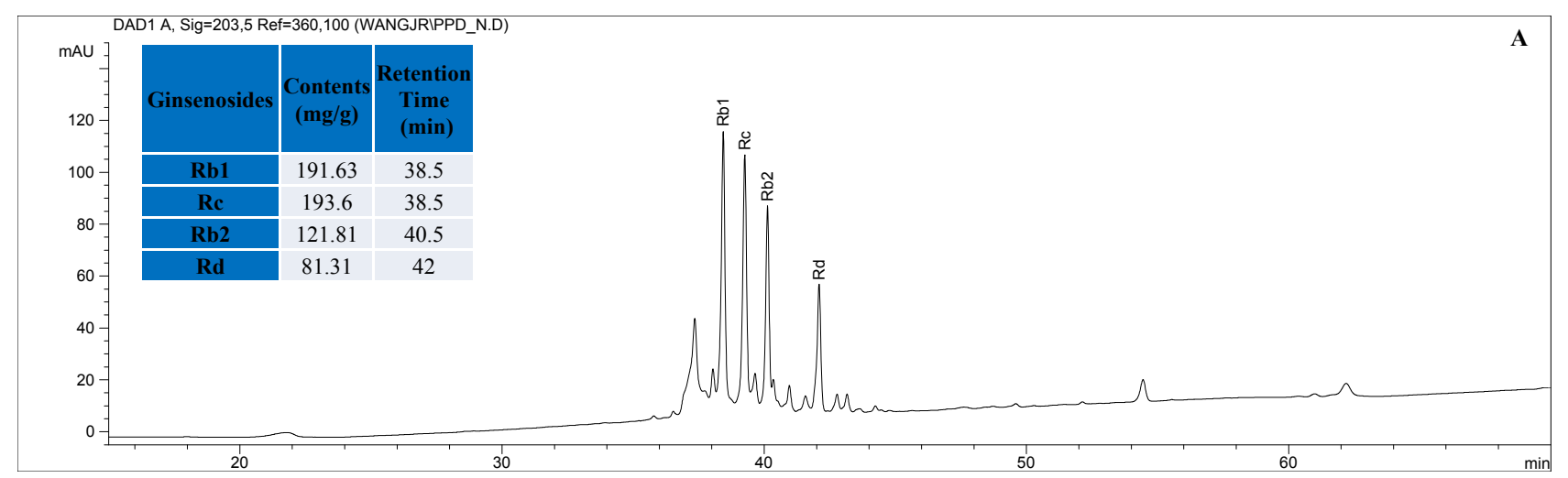


Figure 3. Cont.
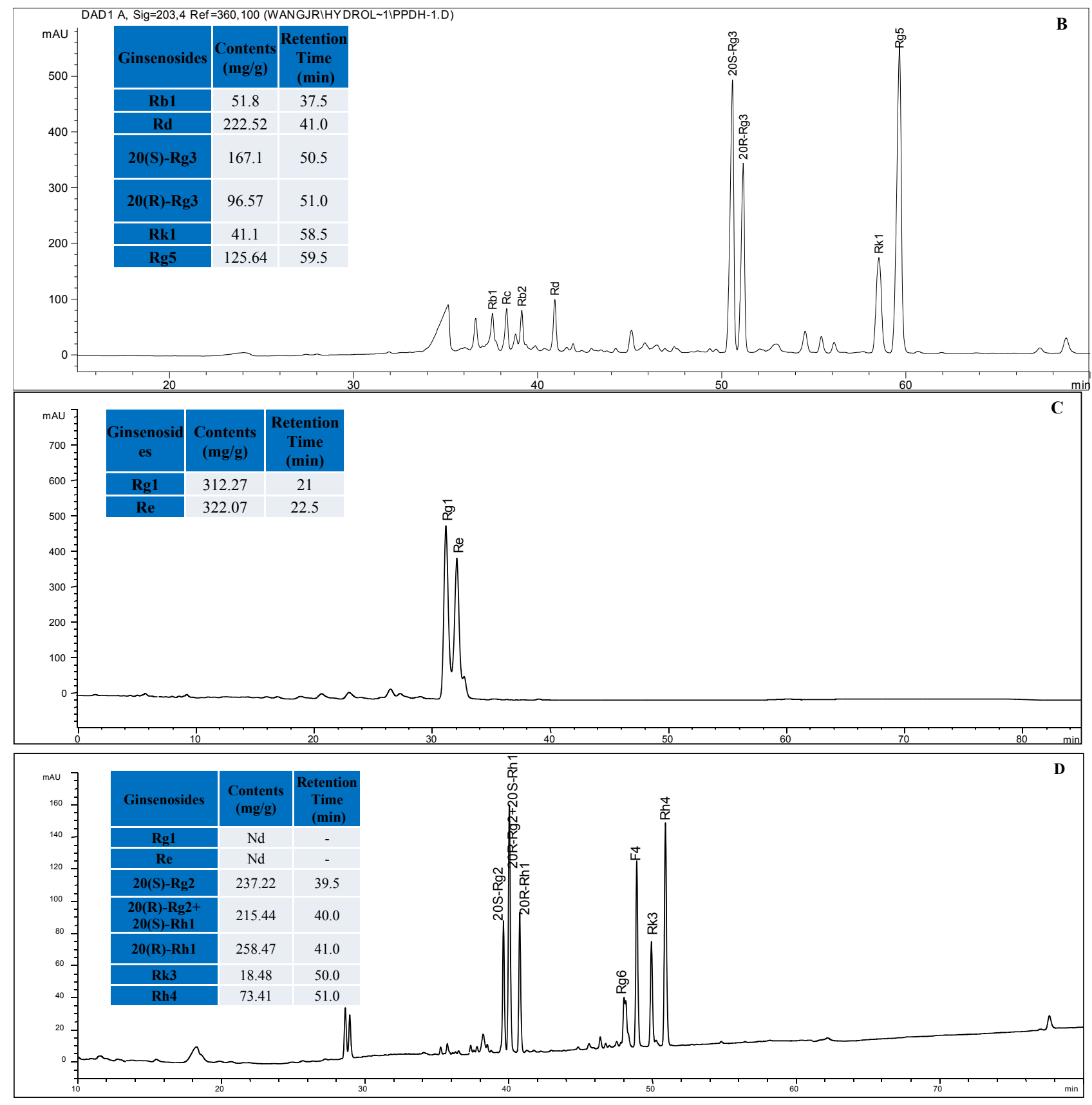

We next examined the cytotoxic potency of the PPDH and PPTH ginsenosides against several cancer cell lines. At the concentrations of $250 \mu \mathrm{g} / \mathrm{mL}$ and $500 \mu \mathrm{g} / \mathrm{mL}$, PPDH ginsenosides were able to reduce the cell survival rates of LLC1 cells at different treatment time periods, but no time-dependent relationship was found (Figure 4).

The $\mathrm{IC}_{50}$ value of the PPD-type ginsenosides fraction decreased from $750 \mu \mathrm{g} / \mathrm{mL}$ (data not shown) down to $180 \mu \mathrm{g} / \mathrm{mL}$ in LLC1 cells after acid hydrolysis. Although the PPT-type ginsenosides fraction displayed no cytotoxic effects, PPTH ginsenosides could reduce cell survival rate of LLC1 cells at a drug concentration of $500 \mu \mathrm{g} / \mathrm{mL}$, but without any time-dependent relationship (Figure 5). 
Figure 4. Time and dose-dependent cytotoxicity of PPD and PPDH on LLC1 cells.
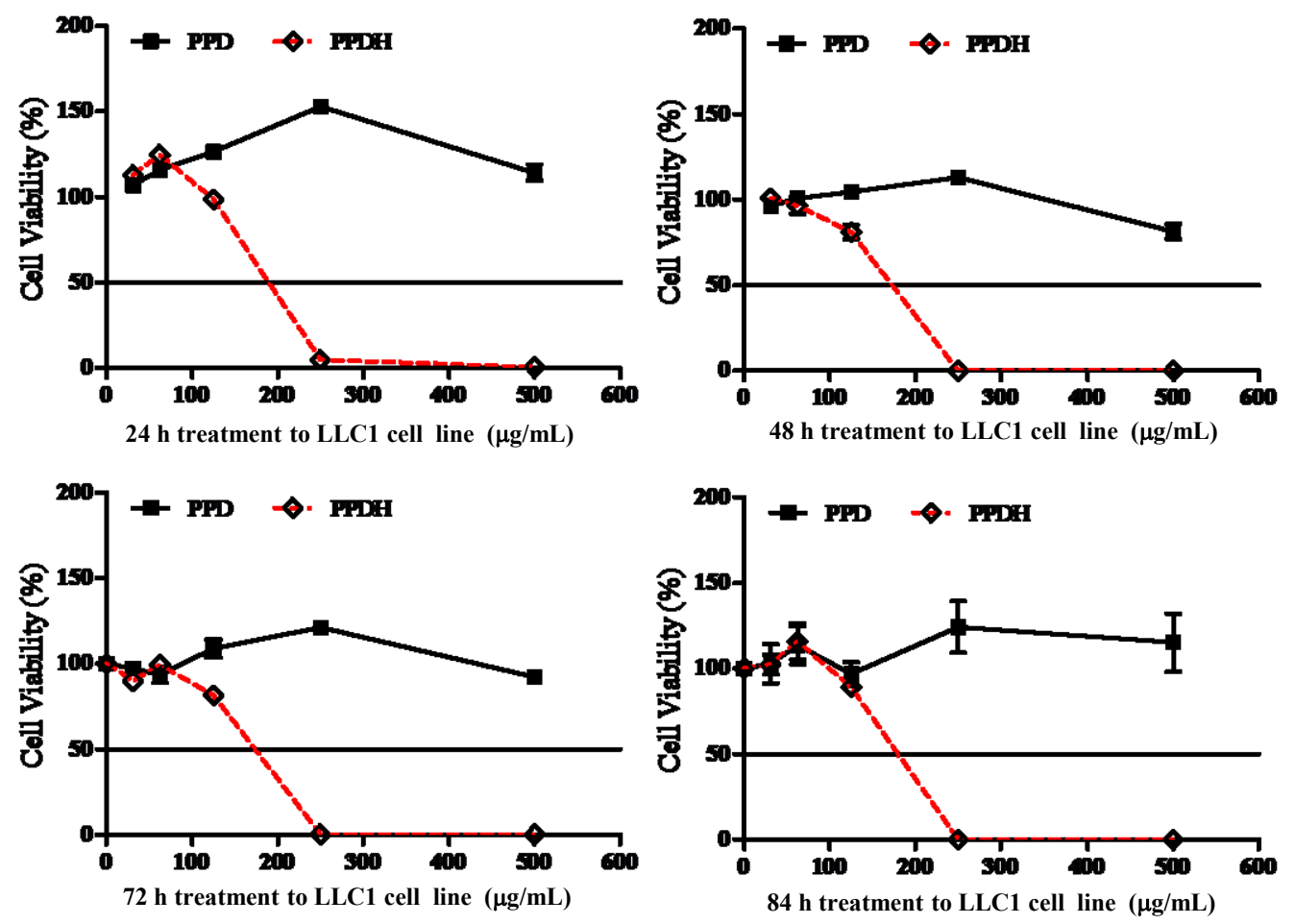

Figure 5. Time- and dose-dependent cytotoxicity of PPT and PPTH on LLC1 cells.
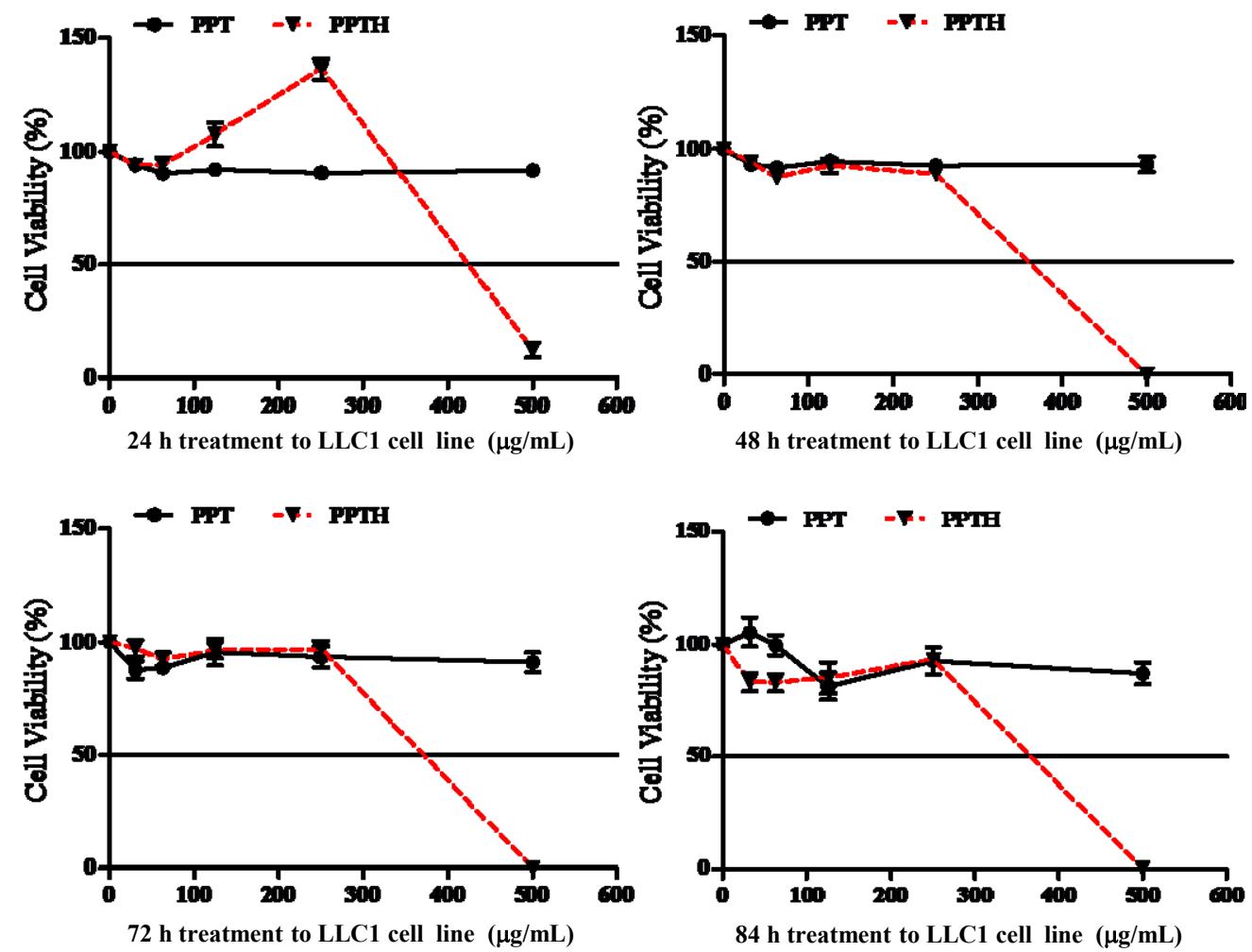

In order to prove whether this cytotoxic effect was specific to tumor cells, we further compared the effect of PPDH and PPTH ginsenosides on cell viability using the LLC1 and lung normal fibroblast cell line, CCD19Lu. The results showed no obvious differences of the cytotoxic potency in PPDHtreated LLC1 and CCD19Lu cells; the $\mathrm{IC}_{50}$ on those two cell lines were $180 \mu \mathrm{g} / \mathrm{mL}$ and $185 \mu \mathrm{g} / \mathrm{mL}$, 
respectively (Figure 6A). Likewise, $\mathrm{IC}_{50}$ values of PPTH ginsenosides on LLC1 and CCD19Lu cells showed no marked differences, i.e., $325 \mu \mathrm{g} / \mathrm{mL}$ and $400 \mu \mathrm{g} / \mathrm{mL}$, respectively (Figure 6B). In addition, as shown in Figures 6C and 6D, PPDH and PPTH ginsenosides displayed similar cytotoxic potencies against four types of cancer cell lines with different genetic backgrounds. Taken together, these results indicate that although the cytotoxic effects of total PPD- and PPT-type ginsenosides fractions were increased after hydrolysis, both almost equally impaired normal lung fibroblasts.

Figure 6. Effect of PPDH or PPTH ginsenosides on inhibition of cell growth of the lung normal fibroblast cell line CCD19Lu and cancerous cell lines.
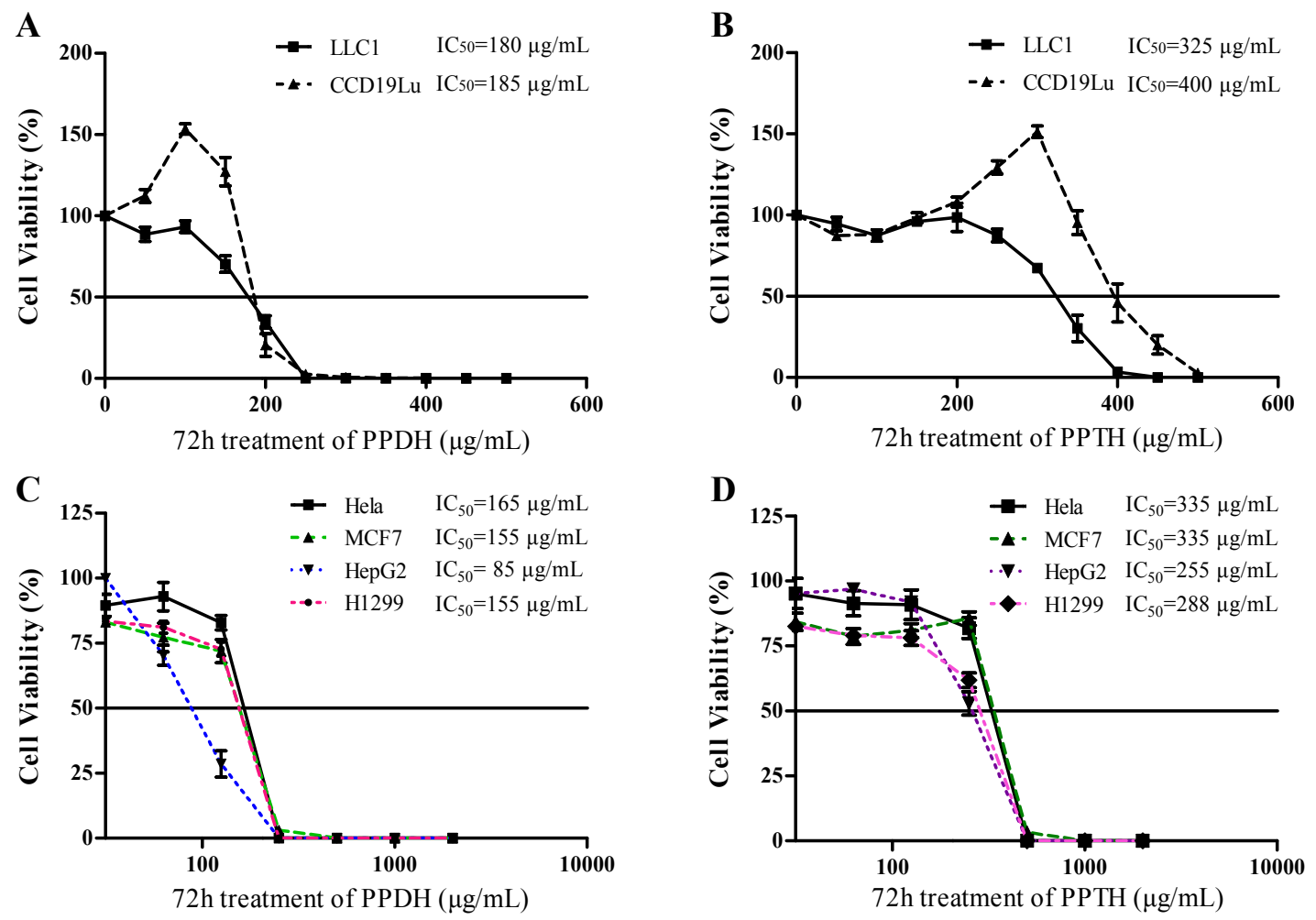

\subsection{Examination of the Cytotoxic Potency of PPD-type Ginsenosides and their Structurally-Modified} PPD-Derivatives

Numerous investigations have revealed that the anticancer activity of ginseng can be predominantly attributed to PPD-type ginsenosides, in comparison to the PPT-type ginsenosides. Accordingly, we examined the cytotoxic activity of PPD-type secondary ginsenosides, including $\mathrm{Rg} 3, \mathrm{Rh} 2$ and the aglycone 20(S)-PPD, in ten cancer cell lines representing seven types of clinical malignancies. 20(S)$\mathrm{Rh} 2$ and $20(R)-\mathrm{Rg} 3$, which are currently marketed as anticancer agent and dietary supplement, respectively, for cancer patients in China, were used as controls. As shown in Figure 7, the cytotoxic potencies of PPD-type ginsenosides showed a descending trend of PPD $>\mathrm{Rh} 2>\mathrm{Rg} 3$, indicating that increase in bioactivity correlated with decrease of the molecular polarity. According to this finding, we speculated that cytotoxic potency might be further enhanced along with increase of the lipophilicity of PPD (1). In order to verify this hypothesis and obtain more potent cytotoxic compounds, two PPDderivatives, 20(S)-dihydroprotopanaxadiol (2) [11,12] and 3 (Figure 8) [13], were synthesized using PPD (1) as raw material. The PPD-derivative 2 was obtained by reducing the double bond in the side 
chain; while the PPD-derivative 3 was obtained by hydrogenation of double bonds after a dehydration reaction at the C-20 position of PPD (1). The cytotoxic activities of these two structurally-modified PPD derivatives were subsequently examined on the same cancer cell lines and compared with PPD (1), Rg3 and Rh2. As demonstrated in Figure 7, 20(S)-Rh2, PPD, 20(S)-dihydroprotopanaxadiol and the PPD-derivative 3, with gradual deglycosylation, hydrogenation and dehydration, exhibited different potencies on inhibition of the cancer cell lines growth in a dose-dependent manner. Using the same drug concentrations of the tested compounds in the experiments, $20(R)-\operatorname{Rh} 2,20(S)-\operatorname{Rg} 3$ and $20(R)-\operatorname{Rg} 3$ showed low cytotoxic potency to the tested cancerous cell lines, although $\operatorname{Rg} 3$ was reported effective for anti-cancer in the in vivo experiments [14-16]. The cytotoxic activity generally occurred in the order of: compound $3>20(S)$-dihydroprotopanaxadiol (2) $>$ PPD (1) $>20(S)$-Rh2 $>20(R)$-Rh2 $\approx 20(R)-\operatorname{Rg} 3 \approx 20(S)-\operatorname{Rg} 3$. For PPD-type ginsenosides, increasing cytotoxic effect was correlated with decrease of the sugar numbers in their chemical structures. As a whole, for PPD derivatives, more lipophilic chemical structures appear to confer more potent cytotoxicity with regard to cancer cells.

Figure 7. Cytotoxicity of PPD-type ginsenosides and PPD-derivatives against various cancer cell lines.

A549

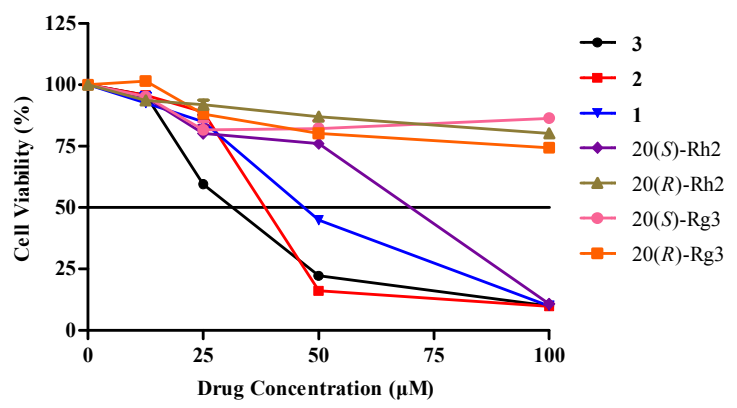

LLC-1

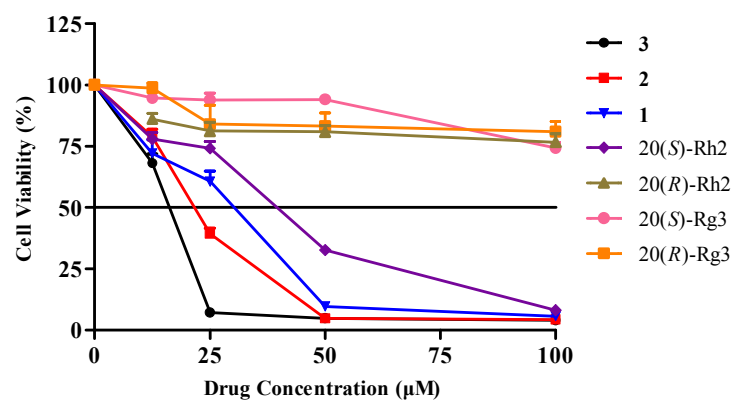

MCF7

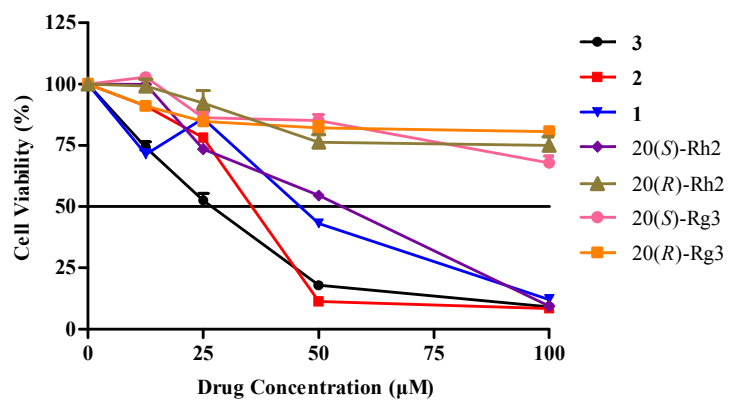

H1299

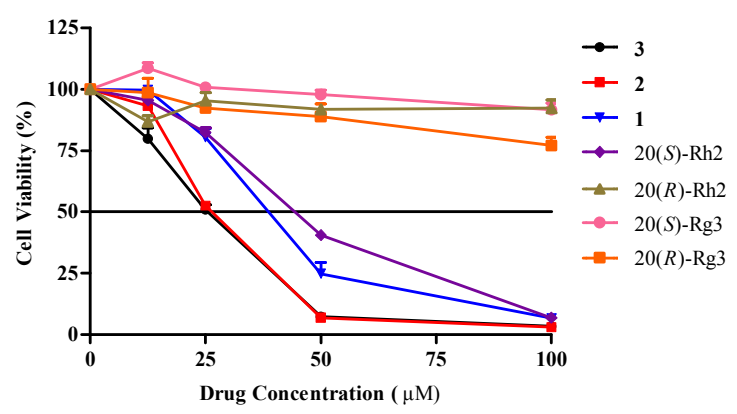

HCT116

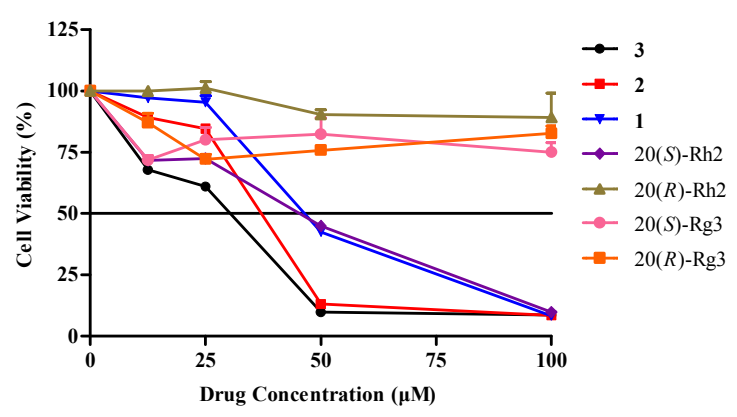

BT474

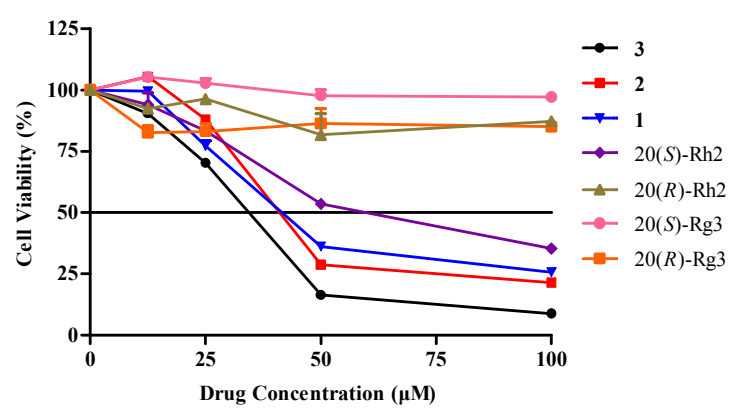


Figure 7. Cont.

LNCaP

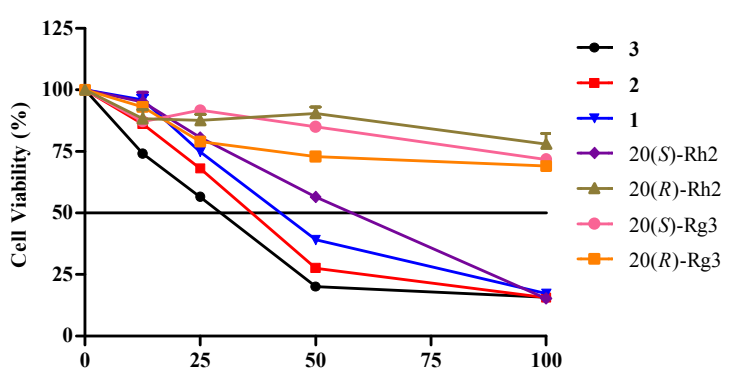

HepG2

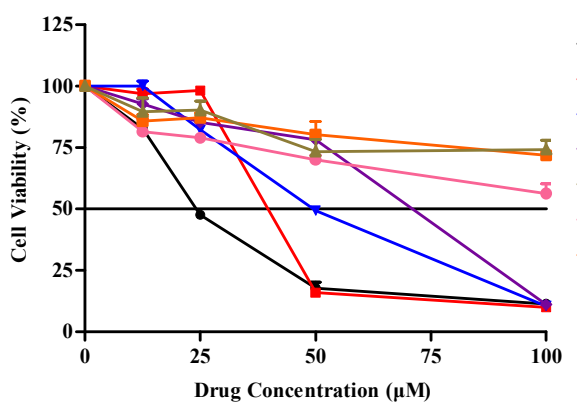

Hela

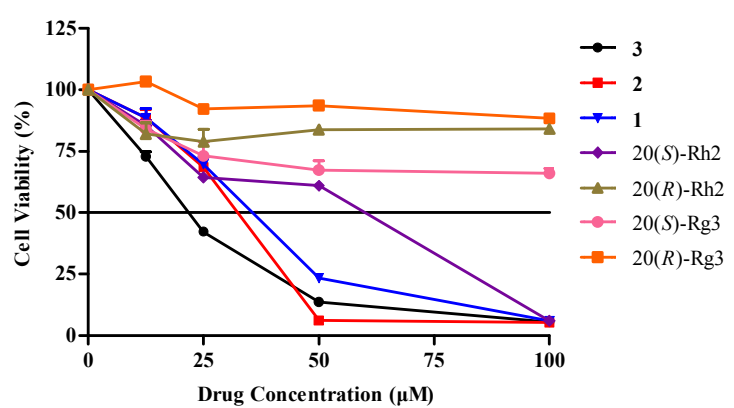

MG-63

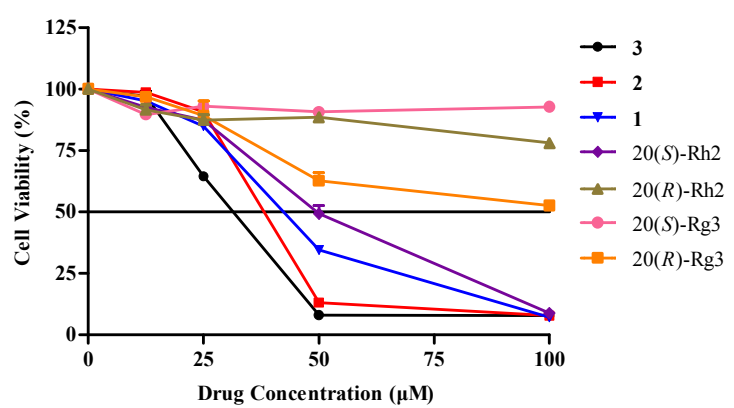

Figure 8. Chemical structures of PPD (1) and its synthetic derivatives 2 and $\mathbf{3}$.<smiles>CC(C)=CCCC(C)(O)C1CC[C@@]2(C)C1C[C@H](O)CC1[C@@]3(C)CC[C@H](O)C(C)(C)C3CC[C@]12C</smiles>

1<smiles>[R]C(C)(CCCC(C)C)C1CC[C@]2(C)C1C(O)C[C@H]1[C@@]3(C)CC[C@H](O)C(C)(C)C3CC[C@]12C</smiles>

2: $\mathrm{R}=\mathrm{OH}$

3: $\mathrm{R}=\mathrm{H}$

\section{Experimental}

\subsection{Instruments and Materials}

${ }^{1} \mathrm{H}-\mathrm{NMR}$ spectra were recorded using a Bruker-400 NMR spectrometer, and chemical shifts are presented in $\delta(\mathrm{ppm})$ relative to $\mathrm{Me}_{4} \mathrm{Si}$ used as internal standard. High resolution mass spectroscopy measurements were performed on a Q-TOF mass spectrometer (Bruker Daltonics, Billerica, MA, USA). 20(S)-Protopanaxadiol was purchased from Shanghai PharmValley Corporation (Shanghai, China).

\subsection{HPLC Analysis of Ginsenosides in RSEH, PPTH and PPDH}

HPLC analyses of ginsenosides in RSEH, PPTH and PPDH were performed on an Agilent (Palo Alto, CA, USA) 1100 series HPLC consisting of a binary pump, an automatic sample injector, and a 
diode array detector. The samples were separated on a Zorbax Eclipse XDB-C18 column (Agilent, $4.6 \mathrm{~mm} \times 250 \mathrm{~mm}, 5 \mu \mathrm{m}$ ) at $25^{\circ} \mathrm{C}$ with a sample injection volume of $20 \mu \mathrm{L}$. The mobile phase was a gradient of water (A) and acetonitrile (B), starting isocratically with $20 \%$ B for 20 min and increasing to $46 \% \mathrm{~B}$ at $20-45 \mathrm{~min}$, then increasing to $55 \% \mathrm{~B}$ at $45-60 \mathrm{~min}$, and finally to $100 \%$ at $60-105 \mathrm{~min}$. The flow rate of the mobile phase was $1.0 \mathrm{~mL} / \mathrm{min}$, and the detector wavelength was $203 \mathrm{~nm}$.

\subsection{Synthesis of the PPD (1) Derivatives $\mathbf{2}$ and $\mathbf{3}$}

20(S)-Dihydroprotopanaxadiol (2): 20(S)-Protopanaxadiol (1, $210 \mathrm{mg})$ in methanol (20 $\mathrm{mL})$ was stirred under a $\mathrm{H}_{2}$ atmosphere at room temperature for $3 \mathrm{hr}$ in the presence of palladium hydroxide on carbon powder $(58 \mathrm{mg})$. The product was purified by ODS column chromatography eluted with a gradient from $80 \%$ aqueous to $100 \%$ methanol to yield PPD-derivative 2 (180 mg). High Resolution ESI-MS (Positive ion mode): $m / z 485.3978[\mathrm{M}+\mathrm{Na}]^{+}$(calculated for $\mathrm{C}_{30} \mathrm{H}_{54} \mathrm{NaO}_{3}$ : 485.3965). ${ }^{1} \mathrm{H}-\mathrm{NMR}$ $\left(\mathrm{CDCl}_{3}\right): 3.60(1 \mathrm{H}, \mathrm{td}, J=10.4,5.2 \mathrm{~Hz}, \mathrm{H}-12), 3.20(1 \mathrm{H}, \mathrm{dd}, J=11.0,4.0 \mathrm{~Hz}, \mathrm{H}-3), 2.04(1 \mathrm{H}, \mathrm{td}$, $J=10.8,7.0 \mathrm{~Hz}, \mathrm{H}-17), 1.18$ (3H, s, H-21), 0.99 (3H, s, H-18), 0.98 (3H, s, H-28), 0.89 (3H, s, H-30), $0.88(6 \mathrm{H}, \mathrm{d}, J=6.3 \mathrm{~Hz}, \mathrm{H}-26$ and H-27), 0.88 (3H, s, H-19), 0.78 (3H, s, H-29), 0.73 (1H, d, $J=10.8 \mathrm{~Hz}, \mathrm{H}-5)$.

Derivative (3) was obtained by the following procedure: $20(S)$-protopanaxadiol $(\mathbf{1}, 500 \mathrm{mg})$ in DMSO $(8 \mathrm{~mL})$ was heated at $140{ }^{\circ} \mathrm{C}$ in an oil bath for $50 \mathrm{~min}$. The reaction solution was directly loaded to an ODS column to give the dehydrated products of PPD (350 mg). The dehydrated products $\left(330 \mathrm{mg}\right.$ ) in methanol $(25 \mathrm{~mL})$ were subjected to catalytic hydrogenation reaction under a $\mathrm{H}_{2}$ atmosphere in the presence of palladium hydroxide on carbon powder $(66 \mathrm{mg})$. The reaction solution was dried and purified over an ODS chromatography column using an gradient from $90 \%$ aqueous to $100 \%$ methanol as eluent to yield compound 3. High Resolution ESI-MS (Positive ion mode): $\mathrm{m} / \mathrm{z}$ $429.4067\left[\mathrm{M}-\mathrm{H}_{2} \mathrm{O}+\mathrm{H}\right]^{+}$(calculated for $\mathrm{C}_{30} \mathrm{H}_{53} \mathrm{O}: 429.4091$ ), $447.4162[\mathrm{M}+\mathrm{H}]^{+}$(calculated for $\mathrm{C}_{30} \mathrm{H}_{55} \mathrm{O}_{2}$ : 447.4197). ${ }^{1} \mathrm{H}-\mathrm{NMR}\left(\mathrm{CDCl}_{3}\right): 3.63(1 \mathrm{H}, \mathrm{td}, J=10.4,5.2 \mathrm{~Hz}, \mathrm{H}-12), 3.20(1 \mathrm{H}, \mathrm{dd}$, $J=11.32,5.0 \mathrm{~Hz}, \mathrm{H}-3), 0.99$ (3H, s, H-18), $0.96(3 \mathrm{H}, \mathrm{s}, \mathrm{H}-28), 0.90,0.80(3 \mathrm{H}, \mathrm{d}, J=6.8 \mathrm{~Hz}, \mathrm{H}-21)$, 0.88 (3H, s, H-30), 0.87 (6H, d, $J=6.8 \mathrm{~Hz}, \mathrm{H}-26$ and H-27), 0.87 (3H, s, H-19), 0.78 (3H, s, H-29), $0.72(1 \mathrm{H}, \mathrm{dd}, J=11.1,1.8 \mathrm{~Hz}, \mathrm{H}-5)$.

\subsection{Cancer Cell Lines and Cell Culture Conditions}

All cancer cells were acquired from the American Type Culture Collection (Rockville, MD, USA) and cultured according to their guidelines.

\subsection{Cytotoxic Activity Assay}

The cytotoxic effects of ginseng extracts, ginsenosides, and their derivatives against cancer cells, expressed as the percentage of cell viability, were determined by MTT assay described previously [17]. The assay was performed using three replicates with three independent experiments. Representative results are showed as means \pm S.E.M., ${ }^{*} p<0.05,{ }^{* *} p<0.01$. 


\section{Conclusions}

Based on our results, the cytotoxic potencies of the hydrolysates of RSE and total PPD-type or PPTtype ginsenosides fraction were much stronger than those of the original RSE and ginsenosides fractions. In particular the PPD-type ginsenosides hydrolysate fraction showed the most potency. Regarding the PPD-type ginsenosides, the cytotoxic potency was generally in a descending order of compound $3>20(S)$-dihydroprotopanaxadiol (2) $>$ PPD $(1)>20(S)-\operatorname{Rh} 2>20(R)-\operatorname{Rh} 2 \approx 20(R)-\operatorname{Rg} 3 \approx$ 20(S)-Rg3. This indicates a clear activity-structure relationship in which less polar compounds possessed higher cytotoxic activity towards the tested cancer cells.

\section{Acknowledgements}

The authors wish to thank Martha A. Dahlen for her kind editing of the English version of this paper.

\section{Conflict of Interest}

The authors declare no conflict of interest.

\section{References and Notes}

1. Ferlay, J.; Shin, H.R.; Bray, F.; Forman, D.; Mathers, C.; Parkin, D.M. Estimates of worldwide burden of cancer in 2008: GLOBOCAN. Int. J. Cancer 2008, 127, 2893-2917.

2. Hernandez, B.Y.; Green, M.D.; Cassel, K.D.; Pobutsky, A.M.; Vu, V.; Wilkens, L.R. Preview of Hawaii Cancer Facts and Figures. Hawaii Med. J. 2010, 69, 223-224.

3. Du, G.J.; Dai, Q.; Williams, S.; Wang, C.Z.; Yuan, C.S. Synthesis of protopanaxadiol derivatives and evaluation of their anticancer activities. Anticancer Drugs 2011, 22, 35-45.

4. Wang, W.; Wang, H.; Rayburn, E.R.; Zhao, Y.; Hill, D.L.; Zhang, R. 20(S)-25-methoxyldammarane-3beta, 12beta, 20-triol, a novel natural product for prostate cancer therapy: Activity in vitro and in vivo and mechanisms of action. Br. J. Cancer 2008, 98, 792-802.

5. Attele, A.S.; Wu, J.A.; Yuan, C.S. Ginseng pharmacology: Multiple constituents and multiple actions. Biochem. Pharmacol. 1999, 58, 1685-1693.

6. Tawab, M.A.; Bahr, U.; Karas, M.; Wurglics, M.; Schubert-Zsilavecz, M. Degradation of ginsenosides in humans after oral administration. Drug Metab. Dispos. 2003, 31, 1065-1071.

7. Pietta, P.; Mauri, P.; Rava, A. Hydrolysis of ginsenosides in artificial gastric fluid monitored by high-performance liquid chromatography. J. Chromatogr. 1986, 362, 291-297.

8. Zhang, X.; Song, F.; Cui, M.; Liu, Z.; Liu, S. Investigation of the hydrolysis of ginsenosides by high performance liquid chromatography-electrospray ionization mass spectrometry. Planta Med. 2007, 73, 1225-1229.

9. Han, B.H.; Park, M.H.; Han, Y.N.; Woo, L.K.; Sankawa, U.; Yahara, S.; Tanaka, O. Degradation of ginseng saponins under mild acidic conditions. Planta Med. 1982, 44, 146-149.

10. Bae, E.A.; Han, M.J.; Kim, E.J.; Kim, D.H. Transformation of ginseng saponins to ginsenoside Rh2 by acids and human intestinal bacteria and biological activities of their transformants. Arch. Pharm. Res. 2004, 27, 61-67. 
11. Usami, Y.; Liu, Y.N.; Lin, A.S.; Shibano, M.; Akiyama, T.; Itokawa, H.; Morris-Natschke, S.L.; Bastow, K.; Kasai, R.; Lee, K.H. Antitumor agents. 261. 20(S)-protopanaxadiol and 20(S)protopanaxatriol as antiangiogenic agents and total assignment of (1)H NMR spectra. J. Nat. Prod. 2008, 71, 478-481.

12. Tanaka, N.; Tanaka, O.; Shibata, S. Chemical studies on the oriental plant drugs. XXVIII. Saponins and sapogenins of ginseng; Stereochemistry of sapogenin of ginsenoside Rb1, Rb2 and Rc. Chem. Pharm. Bull. 1972, 20, 1212-1216.

13. Park, M.H.; Park, T.Y. Treatment and Prevention of Cancer with New Ginsenoside Derivatives. WIPO Patent WO/2005/116042, 7 April 2005.

14. Chen, J.; Peng, H.; Ou-Yang, X.; He, X. Research on the antitumor effect of ginsenoside $\operatorname{Rg} 3$ in B16 melanoma cells. Melanoma Res. 2008, 18, 322-329.

15. Keum, Y.S.; Han, S.S.; Chun, K.S.; Park, K.K.; Park, J.H.; Lee, S.K.; Surh, Y.J. Inhibitory effects of the ginsenoside $\mathrm{Rg} 3$ on phorbol ester-induced cyclooxygenase-2 expression, NF-kappaB activation and tumor promotion. Mutat. Res. 2003, 523-524, 75-85.

16. Yue, P.Y.; Wong, D.Y.; Wu, P.K.; Leung, P.Y.; Mak, N.K.; Yeung, H.W.; Liu, L.; Cai, Z.; Jiang, Z.H.; Fan, T.P.; Wong, R.N. The angiosuppressive effects of 20(R)- ginsenoside Rg3. Biochem. Pharmacol. 2006, 72, 437-445.

17. Wong, V.K.; Cheung, S.S.; Li, T.; Jiang, Z.H.; Wang, J.R.; Dong, H.; Yi, X.Q.; Zhou, H.; Liu, L. Asian ginseng extract inhibits in vitro and in vivo growth of mouse lewis lung carcinoma via modulation of ERK-p53 and NF-kappaB signaling. J. Cell Biochem. 2010, 111, 899-910.

Sample Availability: Samples of the compounds are available from the authors.

(C) 2011 by the authors; licensee MDPI, Basel, Switzerland. This article is an open access article distributed under the terms and conditions of the Creative Commons Attribution license (http://creativecommons.org/licenses/by/3.0/). 